\title{
Synthesis of the Getter for Vacuum Insulation Panels (VIPs) used on Marine Reefer Containers
}

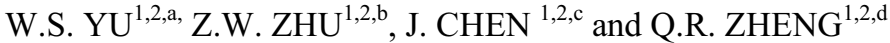 \\ ${ }^{1}$ Provincial Key Laboratory of Naval Architecture \& Ocean Engineering, \\ ${ }^{2}$ Institute of Marine Engineering, Jimei University, Xiamen 361021, China

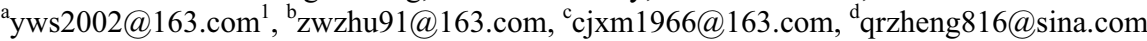

\begin{abstract}
Accurate determination of components released from the VIP is crucial to the selection of the getter that is vital to maintaining its performance of insulation. In this paper, in observation of application of the VIP on marine reefer containers, the VIPs co-developed in our lab were undertaken gas chromatography-mass spectrometer tests to detect the pyrolysis gases released from VIPs at the working condition similar to that of marine reefer containers, and analysis of the composition of the getter was performed by employing the SEM and EDS. It shows that the composite, which was developed by incipient wetness impregnation of cupric nitrate solution on the activated carbon having a high specific surface area, performs well in adsorbing ethylene and propylene from pyrolyzed substances. It is also demonstrated that the as-prepared composite can limit the possibility of hardening of the getter from adsorption of the water vapor. Conclusions are drawn that the getter developed from activated carbon by loading catalytic metal is suitable for VIPs used on marine reefer containers.
\end{abstract}

\section{Introduction}

In recent years, there are about 100000 reefer containers annually constructed and put into use, and the reefer container has become an important role in the international trade [1]. As required by some relevant standards, the refrigeration unit for a reefer container should be able to maintain the temperature within the container about $-18^{\circ} \mathrm{C}-16^{\circ} \mathrm{C}$ where the ambient temperature is about $-30^{\circ} \mathrm{C}-50^{\circ} \mathrm{C}$, and this will also bring about a considerable amount of energy consumption for running the reefer container [2]. As shown in some literatures, the energy consumed by the refrigeration units equipped on reefer containers on board can amount to $60 \%$ of the total energy consumption, energy saving of a reefer container is therefore a necessity for cutting down the running cost and the emission $[3,4]$.

Due to the advantages of the lower thermal conductivity, thin thickness and light weight, vacuum insulation panels(VIPs) have been being used as insulation materials on buildings and refrigerators, and the researches for VIPs on marine reefer containers have already been conducted since 1990s [5]. However, the researches carried out on VIPs for marine purposes are mostly on such aspect as fabrication of core materials and arrangement of the VIPs, and the lithium-barium alloy is unexceptionally selected as a getter for maintaining

* Corresponding author: yws2002@163.com 
the vacuum within the VIPs. Generally speaking, the performance of VIPs mainly depends on the vacuum within the panels, and the selection of the getter should be ensured that the released substances from the core materials can be kept below a limited value via absorbing or adsorbing. Therefore, the getter of VIPs for marine reefer containers should be especially designed as per the physical properties of possibly released gases from core materials under the working conditions similar to those on board a container ship.

To develop a new kind of getter for VIPs used on reefer containers, this paper focuses on three aspects. First, elemental analysis of the getter commercially available was undertaken. Second, the possible ingredients of the released substances from the core materials of VIPs were undergone GC-MS tests. Third, the performance of the getter developed by the activated carbon via surface modification with incipient wetness impregnation of cupric nitrate solution was evaluated.

\section{Experimental}

\subsection{Elemental Analysis of As-prepared Samples}

VIPs was from our cooperator, Fujian Super Tech Advanced Material Co. Ltd. The core of the VIP is composed by silica and glass fiber, Fig. 1 and Table 1 respectively shows the surface appearance and the physical properties of the VIP sample.

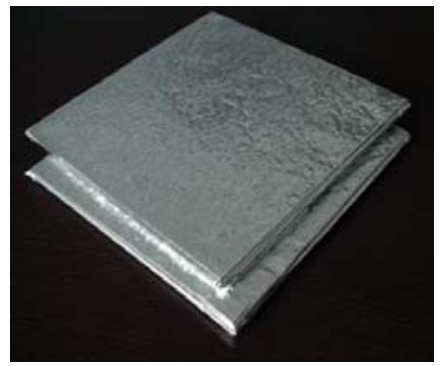

Fig. 1. Pictorial view of the VIPs

Table 1 Physical properties of the vips.

\begin{tabular}{ll}
\hline Item & Value \\
\hline Heat conductivity & $0.0025-0.003 \mathrm{~W} /(\mathrm{m} \cdot \mathrm{K})$ \\
Diameter of the fiber & $2-5 \mu \mathrm{m}$ \\
Dimension & $200 \mathrm{~mm} \times 100 \mathrm{~mm} \times 10 \mathrm{~mm}$ \\
Porosity & $>99 \%$ \\
Volumetric density & $180-250 \mathrm{~kg} / \mathrm{m}^{3}$ \\
\hline
\end{tabular}

For accurately determining the ingredients released from the core materials of VIPs, the selected VIP sample was undergone GC-MS tests under the temperature range from $50^{\circ} \mathrm{C}$ to $290^{\circ} \mathrm{C}$, the results are listed in Table 2, more detailed information can be referred to reference [6]. 
Table 2 The main components released from vips.

\begin{tabular}{cll}
\hline Item & Ingredient & Concentration \\
\hline 1 & 1- chloralkane & $3 \%$ \\
2 & 1,4- dioxane -2,5- dimethyl carbinol & $21.83 \%$ \\
3 & 1- dimethyl- tetramethyl orthosilicate & $41.11 \%$ \\
4 & Diethyl phthalate & $9.99 \%$ \\
5 & 2-t-Butyl-6-[2-hydroxy-2-(2,4,6-trimethylphenyl)ethyl]-[1, & $2.86 \%$ \\
& \\
6 & 3]dioxin-4-one & $4.79 \%$ \\
7 & 13-Hexyloxacyclotridec-10-en-2-one220 & $1.10 \%$ \\
8 & methyl cyclohexylacetate & $10.04 \%$ \\
9 & Butylthiophene & $3.97 \%$ \\
10 & 1,1,2,2-Tetramethylcyclopropane & $1.32 \%$ \\
\hline
\end{tabular}

It can be found in Table 2 that the major component in the released substance is dimethyl- tetramethyl orthosilicate and 1,4-dioxane-2,5-dimethyl carbinol. As per the results in the relevant research, the stabilities of most of alkanes will be weakened by increasing the temperature and will finally be split into ethylene and propylene [7], which should be stabilized by the getter.

As shown in Fig. 2, the getter developed by the co-researcher is in button shape, and the results from SEM and Energy Dispersive Spectrometer (EDS) are listed in Table 3, which reveals that the as-prepared getter is composed by the compound having four elements. Thinking about that the moisture should be a key factor of influencing the vacuum within the VIPs, it can therefore be concluded that the supplied VIPs sample should include $\mathrm{CaCO} 3, \mathrm{CaO}$ and $\mathrm{MgO}$.

Table 3 Results from the analysis of the getter by eds.

\begin{tabular}{ccccc}
\hline Element & $\begin{array}{c}\text { Test1/ } \\
\text { concentration \% }\end{array}$ & $\begin{array}{c}\text { Test2/ } \\
\text { concentration } \%\end{array}$ & $\begin{array}{c}\text { Test3/ } \\
\text { concentration \% }\end{array}$ & Mean value/\% \\
\hline $\mathrm{C}$ & 6.66 & 5.75 & 5.56 & 5.99 \\
$\mathrm{O}$ & 45.95 & 46.95 & 43.87 & 45.59 \\
$\mathrm{Mg}$ & 0.60 & 0.92 & 0.50 & 0.67 \\
$\mathrm{Ca}$ & 46.79 & 46.39 & 50.07 & 47.75 \\
\hline
\end{tabular}

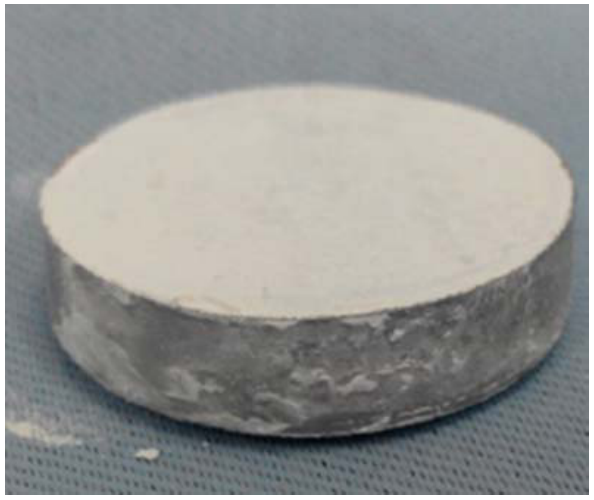

Fig. 2. Pictorial view of the getter for VIPs. 


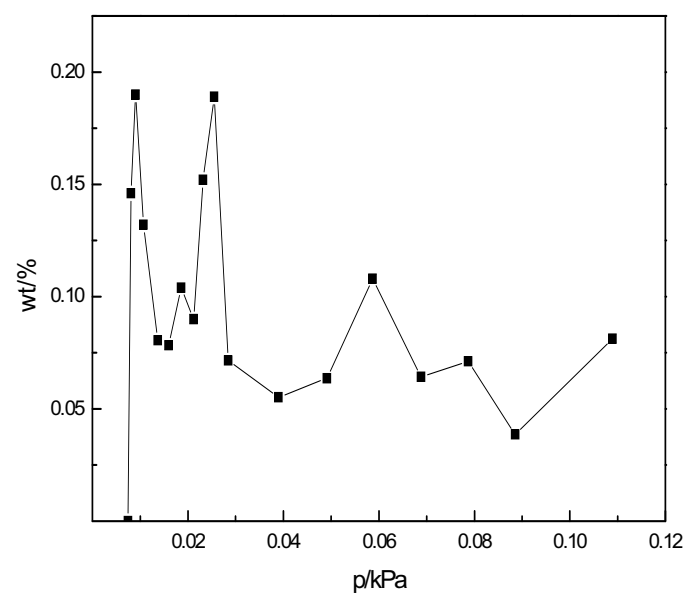

Fig. 3. Isotherm of hydrogen adsorption on the getter at $30^{\circ} \mathrm{C}$.

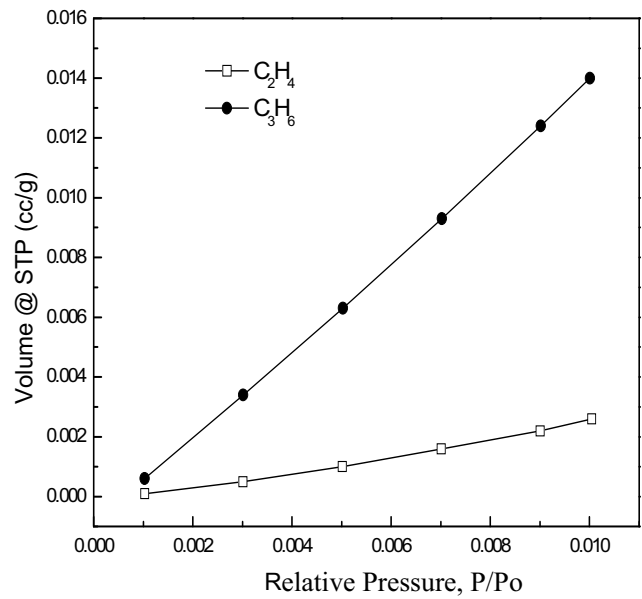

Fig. 4. Isotherms of ethylene and propylene adsorption on the getter at $0^{\circ} \mathrm{C}$.

\subsection{Adsorption Experiments}

In the relevant researches, it demonstrated that hydrogen would also be released from the core materials [8]. Therefore, hydrogen adsorption measurements were firstly carried out on an automated Sieverts apparatus PCTPro E\&E, an instrument especially designed for measuring hydrogen adsorption properties of materials, resulted isotherm is shown in Fig. 3. Quantachrome Autosorb-IQ was employed to measure the isotherms of ethylene and propylene on the getter, results are shown in Fig. 4. It can be found that, in a low pressure region similar to that environment to the getter, the maximum adsorption density of hydrogen on the getter is only about $0.18 \mathrm{wt} \%$, and the adsorption capacity of ethylene and propylene is smaller than $0.014 \mathrm{cc} / \mathrm{g}$. These means that the as-prepared getter is weak in adsorbing/absorbing the gases which may be released from the core materials of VIPs, measures for improving the performance of the getter should therefore be taken.

As mentioned above, the compound that will impair the vacuum of the VIPs should consist of moisture, hydrogen, ethylene and propylene, which should be trapped by the getter and can be remained in a stable state under the conditions similar to those on board a ship. Therefore, the desirable getter should be a composite which can exert an effective attraction to above released substances during the service time of the ship. From our previous studies, it shows that, due to its pore size distribution (PSD), activated carbon is an efficient storage medium for water vapor [9], and the activated carbon having high specific surface area and micro-porous volume is a promising storage medium for hydrogen storage [10]. Here, for comparison studies, we chose commercially available activated carbon SAC-02, silica gel and carbon molecular sieve (CMS) and gravimetrically measured adsorption isotherms of water vapor, results are shown in Fig. 5, the structural parameters of the adsorbents are listed in Table 4, detailed information can be found in reference [9].

It is noted in Fig. 5, where the relative pressure is getting higher than 0.45 , the adsorption amount of vapor on the activated carbon will be larger than those on the silica gel and CMS. Thinking about that the performance of silica gel and CMS can easily be deteriorated where the adsorption has occurred, selection of the activated carbon will be more preferable for vapor adsorption. Meanwhile, the adsorption isotherm of hydrogen on 
the activated carbon was also measured by employing PCTPro E\&E, resulted isotherm is shown in Fig. 6.

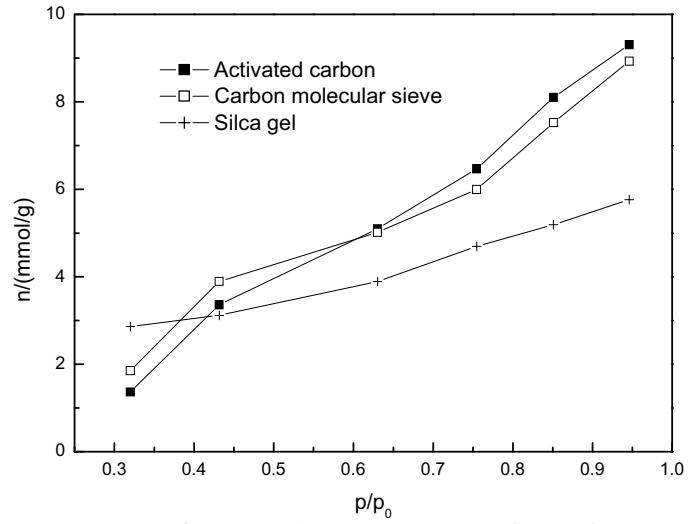

Fig. 5. Isotherms of water vapor adsorption at $30^{\circ} \mathrm{C}$.

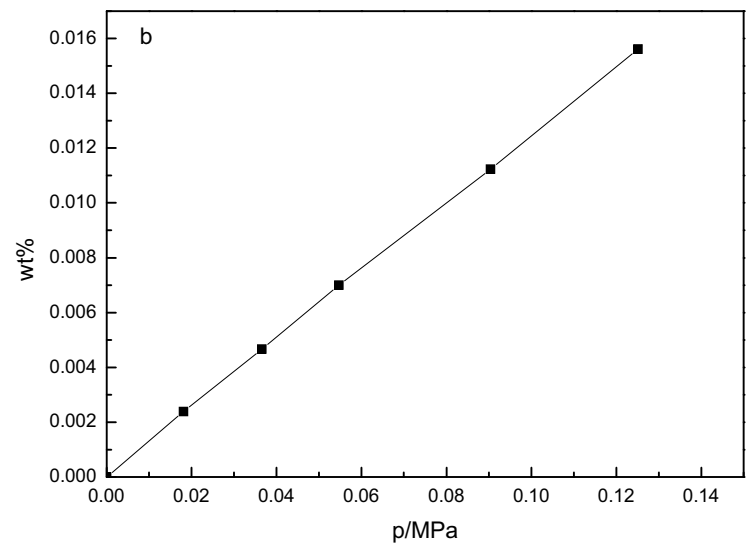

Fig. 6. Isotherm of hydrogen adsorption on the activated carbon at $30^{\circ} \mathrm{C}$.

By comparing Fig. 3 and Fig. 6, one can clearly find that the adsorption amount of hydrogen on the activated carbon is not larger than that of hydrogen on the as-prepared getter, therefore it is a necessity for taking surface modification on the activated carbon.

Table 4 Structural Parameters Of The Adsorbents

\begin{tabular}{ccccc}
\hline Adsorbent & $\begin{array}{c}\text { Apparent } \\
\text { Density } / \mathrm{g} \cdot \mathrm{L}^{-1}\end{array}$ & $\begin{array}{c}\text { BET Specific } \\
\text { Surface } \\
\mathrm{Area} / \mathrm{m}^{2} \cdot \mathrm{g}^{-1}\end{array}$ & $\begin{array}{c}\text { Mesopore } \\
\text { Volume } / \mathrm{mL} \cdot \mathrm{g}^{-1}\end{array}$ & $\begin{array}{c}\text { Micropore } \\
\text { Volume/ } \\
\mathrm{mL} \cdot \mathrm{g}^{-1}\end{array}$ \\
\hline $\begin{array}{c}\text { Activated } \\
\text { carbon }\end{array}$ & 480 & 2074 & 3.05 & 0.25 \\
$\begin{array}{c}\text { Silica gel } \\
\begin{array}{c}\text { Carbon } \\
\text { molecular } \\
\text { sieve }\end{array}\end{array}$ & 870 & 320 & 0.92 & 0 \\
\hline
\end{tabular}




\subsection{Synthesis of the Getter}

As those revealed in literatures, due to the catalytic effect of the transitional metals, the storage capacity of the activated carbon can be greatly enhanced by surface modification through loading the metals $[11,12]$. Here, the activated carbon in 22-40 meshes was firstly impregnated by oxydol for six hours, then undergone incipient wetness impregnation of cupric nitrate solution, finally baked within the muffle furnace at $400^{\circ} \mathrm{C}$ for 4 hours. Adsorption amounts were measured to evaluate the effect of surface modification, results are shown in Fig. 7, more information about the experiment can be referred to [6,9].
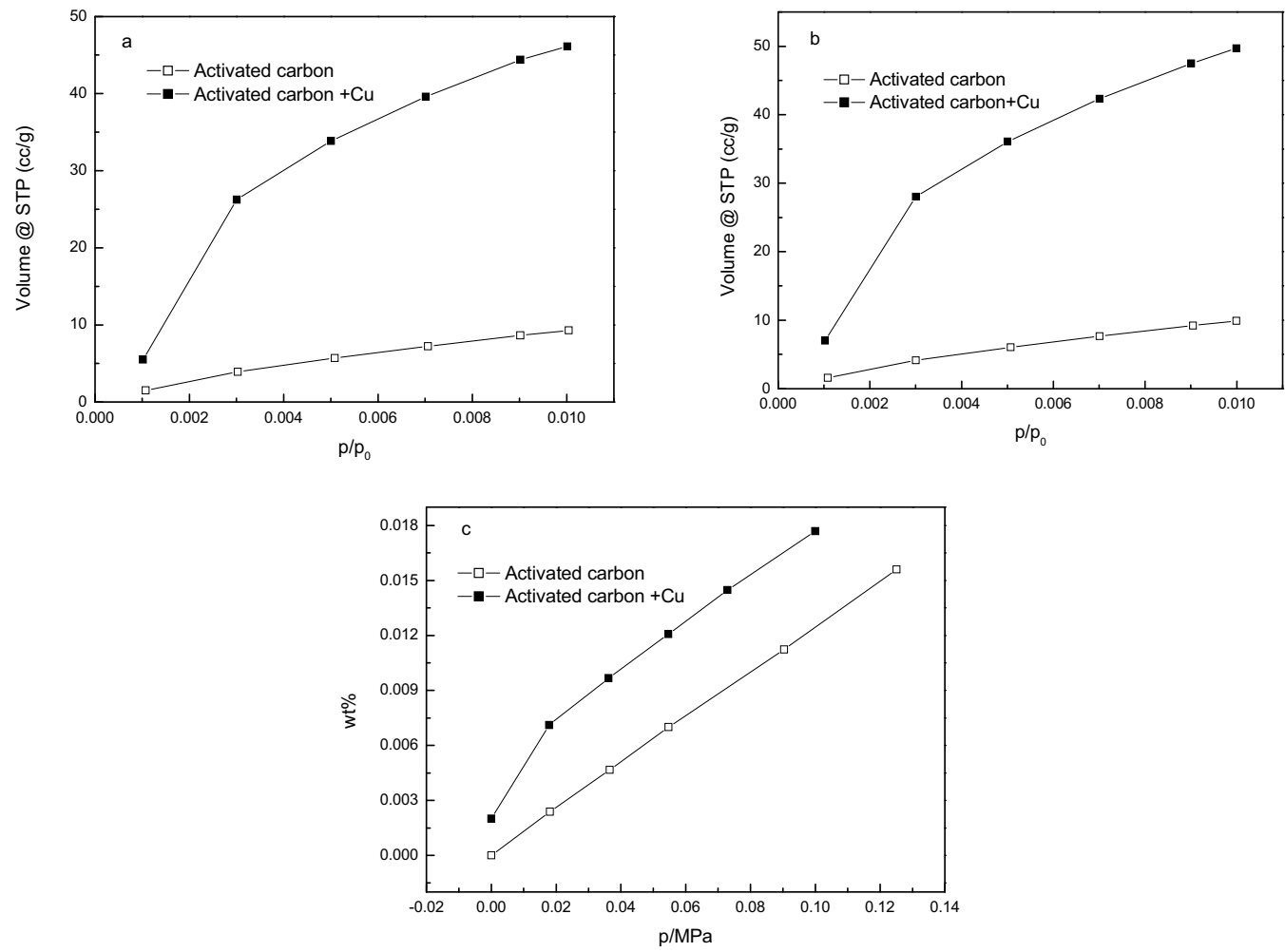

Fig. 7 Isotherms of ethylene(a), propylene(b) and hydrogen adsorption at $30^{\circ} \mathrm{C}$.

From Fig. 7, it can clearly be found that loading cupric ion on the surface of activated carbon can respectively bring about $440 \%, 442 \%$ and $54 \%$ mean increment in ethylene, propylene and hydrogen adsorption.

\section{Conclusions}

To develop a suitable getter for VIPs used on reefer containers, elemental analysis, GC-MS tests and adsorption experiments were conducted on carbon based composite developed by surface modification, and the conclusions obtained can be summarized as follows:

The getter should be specially designed as per the released substances from the core materials of VIPs. The getter containing lithium-barium alloys has little effect on trapping hydrogen and moisture, and the main ingredients emitted from the core materials of VIP are ethylene and propylene. 
Under the similar condition to those on VIPs used on reefer containers, activated carbon having high surface area has a better performance on adsorbing water vapor than silica gel and CMS, and activated carbon is also a promising storage medium for alkene, hydrocarbon and hydrogen.

Surface modification of activated carbon by loading transition metals is an effective way to improve the storage capacity of the activated carbon. Incipient wetness impregnation of cupric nitrate solution on activated carbon can respectively bring about $440 \%, 442 \%$ and $54 \%$ mean increment in ethylene, propylene and hydrogen adsorption.

\section{Acknowledgements}

This research was financially supported by Department of Sciences \& Technology (2015J01216, 2012H0032), Fujian, P.R. China.

\section{References}

1. S.L. Chao, C.C. Chao, Applying a time-space network to reposition reefer containers among major Asian ports, Research in Transportation Business \& Management 17 (2015) 65-72.

2. D. Thijs, C. Paul, V. Pieter, L. O. Umezuruike, N. Bart, Exploring ambient loading of citrus fruit into reefer containers for cooling during marine transport using computational fluid dynamics, Postharvest Biology and Technology 108( 2015)91-101.

3. A.K. Kan, J. Jun, H.D. Han, Research of energy saving on marine reefer containers, Shanghai Ship Building 3(2008)31-33.

4. W.S. Yu, Q. Meng, W. Chen, study on insulation performance of marine refrigerated container's sidewall model embedded with vacuum insulation panels, Ship Engineering 37(2015)85-89.

5. K.H. Brodt, G.C. Bart, Performance of sealed evacuated panels as thermal insulation, International Journal of Refrigeration 17(1994)257-262.

6. Z.W. Zhu, W.S. Yu, X.H. Wnag, W.Z. Luo, Q.R. Zheng, Analysis of adsorptive performance of getters used on a vacuum insulation panel, Journal of Jimei University(Natural Science) 19(2014)446-449.

7. H.M. Zhang, D.Y. Luo, Y.B. Zhao, Z.L. Na, Y.B. Shao, Numerical simulation on distribution of products of typical hydrocarbon molecules pyrolysis, Chemical Reaction Engineering and Technology 27(2011)551-555.

8. X.B. Di, C.G. Bao, Y.M. Gao, Z.G. Xie, Y.N. Hu, On the relationship between thermal conductivity and vacuum of vacuum insulation panels, Vacuum(in chinese) 48(2011)12-15.

9. J. Chen, Research and development of the getter in VIPs for marine reefer containers, Master's thesis, Jimei University of PRC, 2015.

10. Q.R. Zheng, A.Z. Gu, X.S. Lu, W.S. Lin, Study on the adsorption characteristic of hydrogen on the activated carbon at low temperature, Acta Energiae Solaris Sinica 29(2008)613-617.

11. A.D. Lueking, R.T. Yang, Hydrogen spillover to enhance hydrogen storage-study of the effect of carbon physicochemical properties, Appl Catal A Gen 265(2004)259-68.

12. L.F. Wang, C.Y. Yin, R.T. Yang, Selective catalytic reduction of nitric oxide with hydrogen on supported Pd: Enhancement by hydrogen spillover, Appl Catal A Gen 514(2016)35-42. 